\title{
Novel Diagnostic Biomarkers in Liver Disease-a Metabolomic Review
}

\author{
Ahmed I*1, Niaz Z2 ${ }^{2}$ Cheema $S^{3}$, Kassem $\mathbf{W}^{4}$ and Manuele $\mathrm{F}^{5}$ \\ ${ }^{1}$ Department of Medicine, Aldara Hospital and Medical Centre, KSA \\ ${ }^{2}$ Department of Medicine, Mayo Hospital, Pakistan \\ ${ }^{3}$ Department of Surgery, International Medical Centre, KSA \\ ${ }^{4}$ Department of Medicine, Aldara Hospital and Medical Centre, KSA \\ ${ }^{5}$ Department of Medicine, University of Genova, Italy
}

*Corresponding author: Iftikhar Ahmed, Consultant Gastroenterologist Aldara Hospital and Medical Centre, Riyadh KSA and Associate Professor in Al-Faisal University, Yardville Compound | P.O. Box 1105 Riyadh 11431, Kingdome of Saudi Arabia.

To Cite This Article: Iftikhar Ahmed. Novel Diagnostic Biomarkers in Liver Disease-a Metabolomic Review. Am J Biomed Sci \& Res. 2019 - 4(3). AJBSR.MS.ID.000792. DOI: 10.34297/AJBSR.2019.04.000792

Received: : July 03, 2019 | Published: July 29, 2019

\begin{abstract}
Disease of the liver are complex and pose significant challenges to physician due to diagnostic difficulty requiring invasive techniques such as liver biopsy. Changes in the odour of human excretions could provide diagnostic insight into pathophysiological imbalance. Identifying underlying volatiles metabolites responsible for these odorous changes can be correlated with the pathological process within the body. Advances in the technology have enabled us to interpret the volatile signature of these changes in the odour. This has opened a promising area to lay the foundations of a rapid, non-invasive and point of care diagnostic tool. This review explores the diagnostic potential of volatile organic metabolites as novel biomarkers and extends the discussion on the clinical applications of these biomarkers in liver disorders.
\end{abstract}

Keywords: Non-invasive biomarkers; Volatile organic metabolites; Liver disorders, Metabolomics; Gas-chromatography-mass- spectrometer Abbrevations: VOMs: Volatile Organic Metabolites; GC-MS: Gas chromatography Mass spectrometer; GI: Gastrointestinal; SAMe: S-Adenosylmethionine

\section{Introduction}

The liver is one of the vital organs in the human body which plays several critical roles in maintaining human's health and well-being. Its main functions are metabolism of nutrients and excretion of toxic substances, additionally it performs an array of functions that help support immunity, digestion and vitamin storage [1]. The liver is intertwined with nearly every system in the body hence prone to a variety of pathologies. Diseases of the liver are complex and require extensive and often invasive investigations including liver biopsy which remain the currently most reliable test if not the "gold standard' [2]. Liver disorders place a heavy economic burden on healthcare resources and have widespread effect on the individuals, encompassing physical and psychological morbidity and mortality, and poor quality of life [3-5]. There has been an increasing clinical interest at uncovering non-invasive diagnostic tools and scientific research into these has yielded multiple scoring systems, formulae, and imaging modalities to diagnose liver disease and to monitor the disease activity [6,7]. More recently, investigations of volatile metabolites in numbers of research studies have focused on the utilization of these non-invasive biomarkers in the diagnosis of liver disease $[8,9]$.

New developments in the analytical techniques has enabled the detection and interpretation of changes in the volatile organic metabolites (VOMs) to assist diagnosis in liver disorders [10]. VOMs are the chemicals that are products and intermediates of metabolism and may be altered during the diseases process. Changes in the signature of VOMs could potentially provide diagnostic information about health and disease. There are growing number of studies which have reported the differences in VOMs profiles of healthy controls vs. patients with underlying disorders [11-14]. VOMs profiles have been used to segregate different types of liver diseases including stages of liver fibrosis and cirrhosis. The correlation of VOMs with microbiota is interesting and supports the 
hypothesis of gut microbial dysbiosis in the etiology of liver disease. The aim of this paper is to review the diagnostic potential of these volatile biomarkers in different types of liver diseases.

A few complex aetio pathological mechanisms are involved in Liver disorders and clinical presentation may range from an asymptomatic state to complete liver failure depending upon the type and severity of disease $[15,16]$. Due to vital role of liver in various complex metabolic and synthetic functions, any pathological insult to hepatic parenchyma leads to altered concentration of toxic metabolites in the systemic circulation [17]. Some of these metabolites may be exhaled through the lungs giving rise to malodorous breath. Others may be excreted in other biological fluids such as sweat, urine and feces leading to altered odour of these biological excretions [18]. For example, sulphur containing compounds such as dimethyl sulphide, hydrogen sulphide and mercaptans were shown to be increased both in blood as well as in alveolar breath due to incomplete metabolism of sulphur containing amino acids in liver disease. Some of these metabolites may give a characteristic smell to breath; which has a sweet, musty, or even slightly faecal aroma, termed fetor hepaticus [19,20]

\section{Analysis of VOMs in Breath}

Impairment in several metabolic processes in liver was proposed as a cause of these metabolic compounds. Initial studies focused on methionine metabolisms, which in healthy individuals, produced S-adenosylmethionine (SAMe) which regulates hepatocyte growth, differentiation and death. Alteration in the metabolisms of methionine was Studies by Kinsell et al. [21] and later by Chen et al. [22] and found increased levels of sulphur containing compounds in the breath of liver patients in comparison to healthy individuals when both were fed methionine. Similarly, low biosynthesis of $\mathrm{SAMe}$, as a result of impaired methionine metabolism, was also suggested to play a causative role in liver cirrhosis [23]. However, a recent systematic review was unable to demonstrate any significant benefit of SAMe replacement in patients with alcoholic liver disease [24].

Modern and sophisticated analytical techniques completely revolutionized the metabolomic analysis making it possible to detect even very slight alteration in the very small volatile molecules in the breath based on their molecular masses. Consequently, studies from Kaji et al. [25], Tangerman et al. [26] and Hisamura et al. [27] demonstrated the higher levels of these sulphur containing volatiles in the breath of patients with liver disease by using modern analytical methods. More recently Van den Velde and colleagues [28] analyzed the breath from 50 patients with established liver cirrhosis by using GS-MS techniques. In this small study, they found that dimethyl sulphide; acetone; 2-pentanone and 2-butanone were significantly higher in alveolar breath of patients with hepatic cirrhosis. The altered concentration of these metabolites was able to discriminate the cirrhotic group from normal individuals with a sensitivity of $100 \%$ and specificity of $70 \%$.

In another study from Netzer et al. [29], a group of four breath markers were identified with the use of ion molecular reactionmass spectrometry (IMR-MS). Their study group consisted of patients with alcoholic fatty liver disease (AFLD), non-alcoholic fatty liver disease (NAFLD), cirrhosis and healthy controls. Among the detected markers, acetaldehyde, Mx103, isoprene, Mx67 and Mx60 (where Mx indicate unannotated compound mass) were found to discriminate between the diseased groups from healthy control. It is important to note that none of these studies was able to demonstrate whether a disease might have a unique breath volatile pattern.

Several studies support the crucial involvement of oxidative stress in the pathogenesis of liver disease including alcoholic and non-alcoholic hepatotoxicity, infections, iron overload and autoimmune liver damage [30,31]. The peroxidation of polyunsaturated fatty acids, such as linoleic acid and linolenic acid, which are cell membrane components, induces the formation of volatile alkanes that are excreted in the breath. These straight chain aliphatic hydrocarbons have been advocated as noninvasive markers of free-radical induced lipid peroxidation in humans [32,33]. Exhaled hydrocarbons especially breath ethane and pentane, appear to be better correlated with alcohol induced hepatic injury than to other aetiologias.

For example, Letteron et al. [34] measured the ethane levels in the breath of patients with alcoholic and non-alcoholic hepatitis and found it significantly higher in alcohol abuser than other groups, albeit a weak correlation with level of alcohol use, histological scoring or other complications. This stronger correlation of breath ethane with alcohol might be due to increased induction of cytochrome $\mathrm{P} 450$ by alcohol, leading to increased production of oxygen radicals. In contrast to alcoholic induced liver injury where alkanes were the predominant volatiles in breath, in non-alcoholic fatty liver disease (NAFLD), which is more prevalent in obese patients, ethanol levels were found to be raised in exhaled breath of patient even in the absence of ethanol ingestion [35].

The hypothesis of increased endogenous production of ethanol in obese individuals was supported by preliminary animal models followed by human studies showing the role of intestinal microbiota in the production of ethanol in obese patients [36,37]. However, it remains unclear whether increased intestinal permeability with secondary endotoxin-mediated damage, in addition to increased endogenous ethanol, contributes to the development of steatohepatitis in obese individuals [38,39]. A study by Khalid et al [40] studied the breath VOMs profiles of patients with various liver disorders and concluded that patient with different stages of liver disease due to alcohol can be differentiated based on the presence or absence of VOMs fingerprints. Of note in this study, the presence of hepatic encephalopathy was correctly identified in $90.9 \%$ of the alcoholic cirrhosis cohort. In a recent study, Fernandez et al. investigated the breath VOMs of patient with liver cirrhosis before and after liver transplant in comparison with healthy control. They found a group of seven volatiles which were elevated in the breath of patients with cirrhosis versus controls. Following liver transplant, five VOMs out of these (limonene, methanol, 2-pentanone, 2-butanone and carbon disulfide) showed statistically significant decrease in abundance [41]. 


\section{Analysis of VOMs in Blood}

In addition to breath analysis, studies have also reported the analysis of VOMs from blood, which are more representative of the internal environment of biological activities. Goldberg et al. [42] analyzed the serum VOMs of patients with hepatic cirrhosis by using a direct injection capillary column gas chromatography method. They found raised levels of 3-methylbutanal in chronic encephalopathy, which correlated well with severity of the disease. In contrast, Marshall et al. [43] found no difference in the level of 3-methylbutanal in cirrhotic patients when compared to healthy controls. This aldehyde results from breakdown of leucine by bacteria. However, when researchers fed leucine to cirrhotic patients, no change in the clinical conditions were observed even when the value of 3-methylbutanal rose to $700 \%$ above the base level. More recently, a small study published by Ruyi et al. [44] reported the analysis of VOCs in blood of patients with liver cancer. By using the GC-MS technique, they found hexanal; 1-octen-3ol and octane as possible biomarkers of liver cancer with good sensitivity and specificity. However, further studies are needed to evaluate these markers in more details and their correlation with liver cancer.

\section{Conclusion}

In short, quantification of VOCs in alveolar breath and blood as markers for diagnosis and routine monitoring of organic disorders is receiving growing medical interests and appear to fulfil the demand and desire for a means of non-invasive investigation of disease due to its ease of sample collection, repeatability, reproducibility and acceptance by the patients group especially children and younger adults. Investigation of human breath samples with various analytical methods has shown a correlation between patterns of VOCs and the occurrence of certain diseases. Although it has been demonstrated that modern analytical instruments allow the determination of many compounds found in human breath both in normal and anomalous concentrations, but due to technical problems of sampling and analysis, and lack of normalization and standardization, huge variations exist between results of different studies. Consequently, VOCs analysis of breath has yet to be introduced into clinical practice.

Among the more frequently used breath tests are glucosehydrogen breath test for small bowel bacterial overgrowth, hydrogen breath test for lactose intolerance, and the urea breath test to detect Helicobacter pylori infection in the stomach. Breath testing remains an under-valued tool for assessing various gastrointestinal and liver diseases which deserve further attention. This provides an important platform to explore the role of dysbiosis in IBD and other GI disorders pathogenesis and development of novel therapeutic targets. In future, further understanding of faecal VOMs may lead to the development of a rapid and simple point of care diagnosis and monitoring of liver disorders.

\section{References}

1. Ozougwu J (2017) Physiology of the Liver. International Journal of Research in Pharmacy and Biosciences 4(8): 13-24.
2. Bedossa P, Carrat F (2009) Liver biopsy: the best, not the gold standard. J Hepatol 50: 1-3.

3. Stepanova M, De Avila L, Afendy M, Younossi I, Cable R, et al. (2017) Direct and Indirect Economic Burden of Chronic Liver Disease in the United States. Clin Gastroenterol Hepatol 15(5): 759-766.

4. Neff GW, Duncan CW, Schiff ER (2011) The current economic burden of cirrhosis. Gastroenterol Hepatol (N Y) 7(10): 661-671.

5. Williams R, Aspinall R, Bellis M, Camps-Walsh G, Cramp M, et al. (2014) Addressing liver disease in the UK: a blueprint for attaining excellence in health care and reducing premature mortality from lifestyle issues of excess consumption of alcohol, obesity, and viral hepatitis. Lancet 384(9958): 1953-1997.

6. Louvet A, Labreuche J, Artru F, Boursier J, Kim DJ, et al. (2015) Combining data from liver disease scoring systems better predicts outcomes of patients with alcoholic hepatitis. Gastroenterology 149(2): 398-406.

7. Papastergiou V, Tsochatzis EA, Pieri G, Thalassinos E, Dhar A, et al. (2014) Nine scoring models for short-term mortality in alcoholic hepatitis: cross-validation in a biopsy-proven cohort. Aliment Pharmacol Ther 39(7): 721-732.

8. Wilson A (2018) Recent Applications of Electronic-Nose Technologies for the Noninvasive Early Diagnosis of Gastrointestinal Diseases. Proceedings 2: 147

9. Buijck M, Berkhout DJ, de Groot EF, Benninga MA, van der Schee MP, Kneepkens, CM, et al. (2016) Sniffing out paediatric gastrointestinal diseases: The potential of volatile organic compounds as biomarkers for disease. J Pediatr Gastroenterol Nutr 63: 585-591.

10. Shirasu M, Touhara K (2011) The scent of disease: Volatile organic compounds of the human body related to disease and disorder. J Biochem 150: 257-266.

11. Dragonieri S, Schot R, Mertens BJA (2007) An electronic nose in the discrimination of patients with asthma and controls. Journal of Allergy and Clinical Immunology 120: 856-862.

12. Kharitonov SA, Barnes PJ (2002) Biomarkers of some pulmonary diseases in exhaled breath. Biomarkers 7(1):1-32.

13. Phillips M, Cataneo RN, Ditkoff BA, Fisher P, Greenberg J, et al. (2003) Volatile Markers of Breast Cancer in the Breath. The Breast Journal 9(3): 184-191.

14. Phillips M, Boehmer JP, Cataneo RN, et al. (2004) Prediction of heart transplant rejection with a breath test for markers of oxidative stress. The American Journal of Cardiology 94(12): 1593-1594.

15. Chung RT, Stravitz RT, Fontana RJ, Mehal WZ, Reddy KR et al. (2012) Pathogenesis of liver injury in acute liver failure. Gastroenterology 143(3): 1-7.

16. Ramadori G, Moriconi F, Malik I, Dudas J (2008) Physiology and pathophysiology of liver inflammation, damage and repair. J Physiol Pharmacol 59(1): 107-117.

17. Mitchell S, Avesh R, Barrett T, Smith R (1999) Trimethylamine and fetor hepaticus. Scand J Gastroenterol 34(5): 524-528.

18. Shimamoto C, Hirata I, Katsu K (2000) Breath and blood ammonia in Liver cirrhosis. Hepato gastroenterology 47 (32): 443-445.

19. Tangerman A, Meuwese-Arends M, Jansen JMJ (1994) Cause and composition of foetor hepaticus. The Lancet 343(8895): 483.

20. Braden B, Lembcke B, Kuker W (2007) 13C-breath tests: Current state of the art and future directions. Digestive and Liver Disease. 39:795-805.

21. Kinsell LW, Harpet HA, Barton HC, Hutchin ME, Hess JR (1948) Studies in methionine and sulfur metabolism; the fate of intravenously administered methionine, in normal individuals and in patients with liver damage. J Clin Invest 27: 677-688. 
22. Chen S MV, Zieve L (1970) Volatile fatty acids in the breath of patients with cirrhosis of the liver. J Lab Clin Med 75(4): 622-627.

23. Lu SC, Tsukamoto H, Mato JM (2002) Role of abnormal methionine metabolism in alcoholic liver injury. Alcohol 27(3): 155-162.

24. Rambaldi A (2006) S-adenosyl-L-methionine for alcoholic liver diseases. Cochrane Database Syst Rev 8(2): 22-35.

25. Kaji HM, Saito N, Murao M (1978) Evaluation of volatile sulfur compounds in the expired alveolar gas in patients with liver cirrhosis. Clin Chim Acta 85(3): 279-284.

26. Tangerman A, Meuwese-Arends MT, van Tongeren JHM (1983) A new sensitive assay for measuring volatile sulphur compounds in human breath by Tenax trapping and gas chromatography and its application in liver cirrhosis. Clinica Chimica Acta 130(1): 103-110.

27. M Hisamura (1979) Quantitative analysis of methyl mercaptan and dimethyl sulfide in human expired alveolar gas and its clinica application: study in normal subjects and patients with liver diseases. Nippon Naika Gakkai Zasshi 68(10): 1284-1292.

28. Van den Velde S, Nevens F, Van Hee P, van Steenberghe D, Quirynen M (2008) GC-MS analysis of breath odor compounds in liver patients. J Chromatogr B Analyt Technol Biomed Life Sci 875(2): 344-348.

29. Netzer M, Millonig G, Osl M, Pfeifer B, Praun S, et al. (2009) A new ensemble-based algorithm for identifying breath gas marker candidates in liver disease using ion molecule reaction mass spectrometry. Bioinformatics 25(7): 941-947.

30. Parola M, Robino G (2001) Oxidative stress-related molecules and liver fibrosis. Journal of Hepatology 35(2): 297-306.

31. Nitti M, Pronzato MA, Marinari UM, Domenicotti C (2007) PKC signaling in oxidative hepatic damage. Molecular Aspects of Medicine 29(1-2): 3642.

32. Kneepkens CM LG, Roy CC (1994) The potential of the hydrocarbon breath test as a measure of lipid peroxidation. Free Radic Biol Med 17(2): 127-160.

33. Van Gossum A (1989) Breath alkanes as an index of lipid peroxidation. Eur Respir J 2: 787-791.
34. Lettéron P, Berson A, Fromenty B, Fisch C, Degott C, et al. (1993) Increased ethane exhalation, an in vivo index of lipid peroxidation, in alcohol-abusers. Gut 34(40): 409-414.

35. Nair S, Cope K, Terence RH (2001) Obesity and female gender increase breath ethanol concentration: potential implications for the pathogenesis of nonalcoholic steatohepatitis. Am J Gastroenterol 96: 1200-1204.

36. Cope K, Risby T, Diehl AM (2000) Increased Gastrointestinal Ethanol Production in Obese Mice: Implications for Fatty Liver Disease Pathogenesis. Gastroenterology 119:1340-1347.

37. Coldwell BB (1965) A note on the estimation and disappearance of alcohol in blood, breath and urine from obese and diabetic patients. J Forensic Sci 10: 480-489.

38. Koteish A, Mae Diehl A (2002) Animal models of steatohepatitis. Best Practice \& Research Clinical Gastroenterology 16: 679-690.

39. Yang SQ, Lin HZ, Lane MD, Clemens M, Diehi A (1997) Obesity increases sensitivity to endotoxin liver injury: Implications for the pathogenesis of steatohepatitis 94(6): 2557-2562.

40. Khalid TY, De Lacy Costello B, Ewen R, White P, Steven S, et al. (2013) Breath volatile analysis from patients diagnosed with harmful drinking cirrhosis and hepatic encephalopathy: a pilot study. Metabolomics 9: 938-948.

41. Fernández Del Río R, O’Hara ME, Holt A (2015) Volatile Biomarkers in Breath Associated with Liver Cirrhosis-Comparisons of Pre- and Postliver Transplant Breath Samples. EBiomedicine 2(9): 1243-1250.

42. Goldberg EM BL, Sandler S (1981) A gas chromatographic-mass spectrometric study of profiles of volatile metabolites in hepatic encephalopathy. J Chromatogr 226(2): 291-299.

43. Marshall AW DM, Morgan MY (1985) Plasma 3-methylbutanal in man and its relationship to hepatic encephalopathy. Clin Physiol 5(1): 53-62.

44. Ruyi X, Ling D, Si Z, Chunhui D, Taotao L, et al. (2008) Investigation of volatile biomarkers in liver cancer blood using solid-phase microextraction and gas chromatography and mass spectrometry. Rapid Commun Mass Spectrom 22(8): 1181-1186. 